\title{
SEQUENTIAL CHEMICAL FRACTIONATION AS A TOOL FOR ACCESSING THE ORGANIC MATTER IN BIOCHAR
}

\author{
${ }^{1}$ David ŠIRU゚ČEK, ${ }^{1}$ Michal KALINA, ${ }^{1}$ Martina KLUČÁKOVÁ \\ ${ }^{1}$ Brno University of Technology, Faculty of Chemistry, Brno, Czech Republic, EU
}

https://doi.org/10.37904/nanocon.2021.4372

\begin{abstract}
This contribution is focused on utilization of a sequential chemical fractionation method for determination of organic matter content, its distribution in biochar and the possible use of biochar as a soil conditioner in agriculture. For these purposes the optimized chemical fractionation procedure was used to reveal the content of organic matter in different biochar samples with different properties (samples with European biochar certification for usage in agriculture) which depend significantly on the temperature used during a pyrolysis of biomass residues in the process of biochar production. In parallel, these samples were also fractionated by classic alkaline extraction to obtain the so-called extractable fraction of organic matter (NOM).

Biochar is one of the important soil conditioners, known for having positive effect on crop yield, soil quality, nutrient cycle and carbon sequestration due to the transfer of organic carbon from it to the soil. However, the effect depends on the properties of the biochar, its doses to the soil, but also on the properties of the soil itself. Surprisingly, some authors point to the fact that biochar does not always have a positive effect on soil, plants or microfauna. Therefore, it is necessary to perform its depth characterization to be able to predicts its role in soil and its optimal application dose.

Individual fractions obtained from sequential chemical fractionation as well as NOM samples were characterized by methods of elemental analysis (determination of organic elements content), thermogravimetry (contents of ash, organic matter and moisture). The results showed that the method of sequential chemical fractionation gives higher yields of organic matter compared to classical alkaline extraction. Moreover, the obtained fractions divided according to solubility and strength of binding to residual inorganic matter of biochar can be better characterized by physical-chemical methods, which provides more detailed information about organic matter in biochar and its possible effect on soil properties.
\end{abstract}

Keywords: Biochar, organic matter, chemical fractionation, elemental analysis, thermogravimetric analysis

\section{INTRODUCTION}

Soil organic matter (SOM) is one of the soil components consisting of three basic types - living organisms (worms, insects, microorganisms), dead microorganisms and finally matter formed from organic residues of plants, animals and humus. The decomposition of the first two types creates nutrients for plants (nitrogen, potassium, phosphorus) [1-3]. The main reactive part of SOM is humic substances - natural substances arising from the chemical and biological degradation of plants and animal remains. They are crucial in ecosystems because they regulate global carbon and nitrogen cycles, the growth of plants and microorganisms, the transport of anthropogenic compounds and heavy metals, and the stabilization of soil structure. To date, however, no technologies have been developed to control the activity of humic substances due to their high molecular complexity. There is a broad variety of analytical techniques for their characterization, however, none of these techniques alone is sufficient to elucidate the structural complexity of particles of humic molecules $[4,5]$. 
In general, humic substances are supramolecular associations of heterogeneous, relatively small molecules in nanoscale range that are held together by weak non-covalent interactions, such as hydrogen bonds and hydrophobic forces. The complexity of the humic molecule can be reduced by the gradual destruction of intramolecular interactions that stabilize these complex suprastructures (composed of nanoparticles of organic matter) and thus individual fractions of colloidal size of humic substances (organic matter) can be isolated. These individual fractions of organic matter obtained from sequential fractionation are called humeomics. It is a gradual separation of molecules from humic suprastructures by controlled cleavage of intermolecular bonds. In terms of particle size, these particles obtained by sequential fractionation are colloidal in size which significantly improve the resolution of various analytical methods often used for characterization $[6,7]$.

In recent years, increasing demands have been placed on crop yields which also brings with it increased demands on soil fertility. For these reasons, various substances are often used to increase fertility, which are added artificially. They are most often fertilizers. Recently, however, research has also focused on the application of alternative additives to soils in order not only to increase fertility but also to improve the physicochemical properties of the soil and to increase the content and stabilization of soil organic matter. These additives are often included in a group called - soil supportive substances (SSS). Substances that are widely used for application include, for example, lignite fly ash, biochar and a mixture of fractions of humic substances. It is also very important to apply a suitable form with a given particle size, internal structure and morphology (porous structure) [8,9].

As mentioned before, one of the soil supportive substances is a biochar which is kind of a charcoal mostly produced by pyrolysis of biomass (any substance that has an organic character and comes from plants and animals) in absence of oxygen. Biomass can be considered an excellent source material, as it is renewable and includes many things that are often considered waste. It is a stable, solid and carbon rich substance that can endure in soil for thousands of years. It is characterized by its physical and chemical properties, such as adsorption capacity or composition which vary depending on the starting material and production conditions (temperature). Its main benefit can be found in the agricultural sector where it is used as a soil supplement which significantly improves soil quality. Besides its effect on physico-chemical properties of soil (soil structure, compactness, water and ion sorption capacity, $\mathrm{pH}$, conductivity) $\mathrm{tt}$ contributes to the sustainability of agriculture and makes a positive influence to the transfer of atmospheric carbon to the soil which has a positive impact on flora, water and air $[10,11]$.

\section{EXPERIMENTAL}

\subsection{Materials and Methods}

For this purpose, a sample of biochar from the Czech company BIOUHEL.CZ s.r.o. was used. But before it could be used, large particles and other impurities (incompletely pyrolyzed biomass or tiny stones) were removed from the material using a sieve. For study purposes, the method of sequential chemical fractionation was used in the work. Organic matter contained in biochar affects the key properties of the soil such as fertility, ability to retain water, transport nutrients etc. In order to be able to describe the effect of biochar, it is necessary to precisely characterize the organic matter which must first be separated from the inorganic part. In order to do that, the method previously mentioned - sequential chemical fractionation - was performed and for comparison purposes, the classical procedure - alkaline extraction - was performed too. Using the sequential chemical fractionation allowed us to separate all the different-strongly bound fractions of similar types of molecules. The alkaline extraction only allowed us to see a mixture of organic molecules contained but overall, this method wasn't really good to use in order to separate the organic matter from biochar. Three replicates of sequential chemical fractionation were performed for each of these three samples. 


\subsection{Sequential Chemical Fractionation}

The procedure of sequential chemical fractionation was adopted from the publications of Nebbioso and Drossos [6,7]. The details of the optimization of this chemical extraction procedure of organic matter for the samples of soil and lignite are summarized in chapters 2.2.1 - 2.3 and can be found in our previous publication [12].

\subsubsection{Water soluble fraction 1 (AQU1)}

First step was to dry used organic material in an oven at $45{ }^{\circ} \mathrm{C}$ to evaporate all the water. Then $50 \mathrm{~g}$ of dried material was transferred to Erlenmeyer flask and $150 \mathrm{ml}$ of $0,1 \mathrm{M} \mathrm{HCl}$ was added. Flasks were covered with a double layer of parafilm and left on a shaker overnight. The next day samples were filtered under reduced pressure. Filtrate was frozen and all the water was evaporated in a freeze drier.

\subsubsection{Organic soluble fraction 1 (ORG1)}

Dried solid material after AQU1 was mixed with $100 \mathrm{ml} \mathrm{DCM}$ and $50 \mathrm{ml} \mathrm{MeOH}$ in Erlenmeyer flask and covered with a double layer of aluminium foil and left on a shaker overnight. The next day samples were filtered under reduced pressure. All the solvent was evaporated on a rotary evaporator at $50^{\circ} \mathrm{C}$. The residue after evaporation was transferred to vials left to evaporate the solvent.

\subsubsection{Water and organic soluble fractions 2 (AQU2 and ORG2)}

Dried solid material after ORG1 separation was transferred to high-pressure sealable teflon flasks and $50 \mathrm{ml}$ of a $12 \%$ solution of boron trifluoride in methanol was added. Vials were tightly sealed, clamped in a metal rack and placed in an oven at $85{ }^{\circ} \mathrm{C}$ for 8 hours. Then vials were removed from the oven, let to cool down and additional $50 \mathrm{ml}$ of the solution was added. Vials were sealed and clamped again and placed in the oven for another 8 hours under same conditions. Then after cooling down the solution was filtered under normal pressure and the solid material was dried. The filtrates were L/L extracted (liquid to liquid) with chloroform. The obtained AQU2 and ORG2 fractions were purified and dried in the same way as was described for AQU1 and ORG1.

\subsubsection{Water and organic soluble fractions 3 (AQU3 and ORG3)}

Dried solid material after the previous step was covered with $50 \mathrm{ml}$ of $1 \mathrm{M}$ solution of $\mathrm{KOH}$ in $\mathrm{MeOH}$ (for the lignite sample a $100 \mathrm{ml}$ of the solution was used). A magnetic stirrer was added, the sample flask was attached to a direct condenser and heater was set to $75^{\circ} \mathrm{C}$. The mixture was refluxed under nitrogen atmosphere for 2 hours. Then all the content in the flask was filtrated under reduced pressure and the solid material was dried. The filtrates were extracted with dichloromethane using L/L extraction. The obtained AQU3 and ORG3 fractions were purified and dried in the same way as was described for AQU1 and ORG1.

\subsubsection{Water soluble fraction 4 (AQU4)}

Dried solid material after the previous step was covered with $50 \mathrm{ml}$ of a $47 \%$ solution of $\mathrm{HI}$ in water (for the lignite sample a $100 \mathrm{ml}$ of the solution was used). A magnetic stirrer was added, the sample flask was attached to a direct condenser and heater was set to $75^{\circ} \mathrm{C}$. The mixture was refluxed under nitrogen atmosphere for 2 days. Then all the content in the flask was filtrated under reduced pressure and the solid material was dried. The filtrates were neutralized with $\mathrm{NaHCO}_{3}$ to $\mathrm{pH} 7$ and the iodine contained was reduced with $\mathrm{Na}_{2} \mathrm{~S}_{2} \mathrm{O}_{3}$. Then the filtrates were dialyzed against distilled water and freeze-dried.

\subsubsection{Residual extractable organic matter fraction (ResOM)}

To isolate the residual organic matter, $5 \mathrm{~g}$ of dried solid material after AQU4 fractionation and $45 \mathrm{ml}$ of extractant $\left(0,5 \mathrm{M} \mathrm{NaOH}\right.$ and $\left.0,1 \mathrm{M} \mathrm{Na}_{4} \mathrm{P}_{2} \mathrm{O}_{7}\right)$ were mixed. Samples were left on a shaker overnight. The samples were filtered and the filtrates were dialyzed against distilled water and freeze-dried. 


\subsection{Natural organic matter fraction isolation (NOM)}

In parallel, a conventional alkaline extraction was performed. Thus $5 \mathrm{~g}$ of the prime material was covered with $45 \mathrm{ml}$ of extractant $\left(0,5 \mathrm{M} \mathrm{NaOH}\right.$ and $\left.0,1 \mathrm{M} \mathrm{Na}_{4} \mathrm{P}_{2} \mathrm{O}_{7}\right)$. After overnight shaking, the samples were filtered under reducer pressure and the filtrates were dialyzed against distilled water and freeze-dried.

\section{RESULTS AND DISCUSSION}

\subsection{Yield comparison depending on the method used}

As mentioned before, yields of extractable organic matter from biochar were obtained using two methods sequential chemical fractionation (humeomics) and alkaline extraction. Based on the obtained yields (Figure 1) it can be said that the largest fraction is AQU4, however, a majority of it are $\mathrm{NaHCO}_{3}$ and $\mathrm{Na}_{2} \mathrm{~S}_{2} \mathrm{O}_{3}$ used during the fractionation. On the other hand, the AQU1 fraction, which consists of unbound or only weakly bound molecules, is significantly higher than other samples obtained. A similar trend can be observed with the ORG (organo-soluble) samples. Comparing the humeomics ( $\mathrm{HHUMEO)}$ ) and the alkaline extraction (AE) we notice that $A E$ yield is several times smaller but still we have to take in account the elemental analysis and thermogravimetry data to calculate the organic matter content which is much more significant and eliminates the content of salts introduced into the individual fractions during the fractionation procedures.

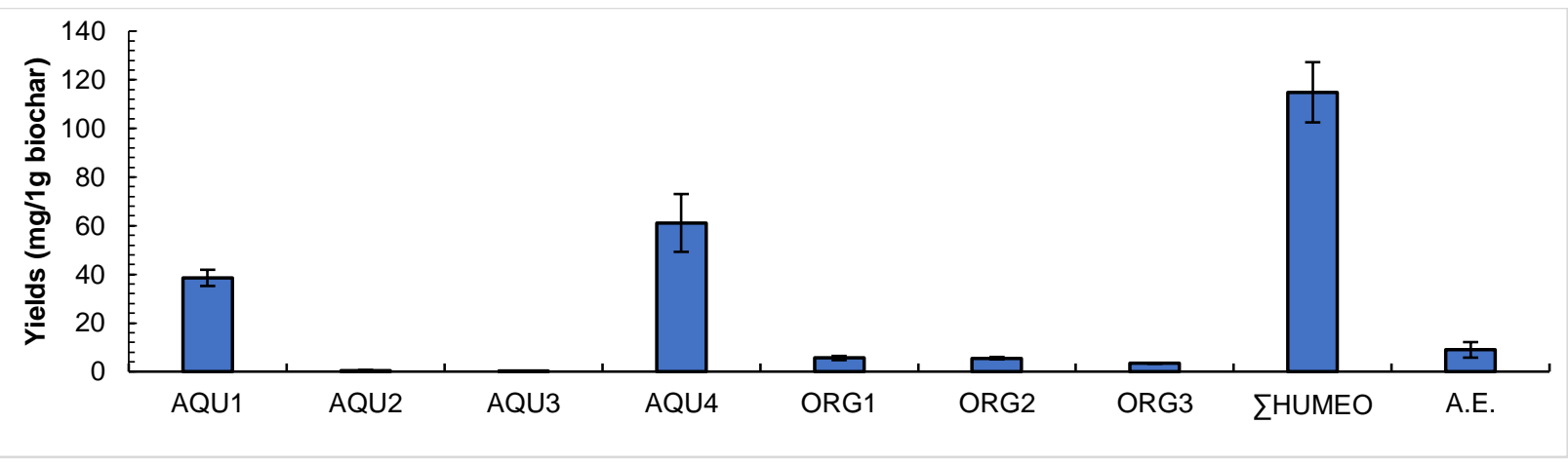

Figure 1 Average yields obtained by both sequential chemical fractionation and alkaline extraction related to $1 \mathrm{~g}$ of biochar used during both processes.

\subsection{Elemental analysis and thermogravimetry application}

Using elemental analyses, the content of carbon, hydrogen, nitrogen and sulfur was determined and the content of oxygen could be later calculated after the determination of moisture and ash content using thermogravimetry. Here we only focus on the carbon and nitrogen content as these are the most important in connection with the application of biochar to soil to improve its fertility and improve conditions for growth. The source biochar sample was also subjected to elemental analysis in order to determine the content or carbon and nitrogen. The total amount of organic carbon contained in biochar was (476 \pm 50$) \mathrm{mg}$ and nitrogen was (26 \pm 4$) \mathrm{mg}$ per $1 \mathrm{~g}$ of biochar. Using the thermogravimetry data shows that the sample of biochar used contains approximately $(699 \pm 45) \mathrm{mg}$ per $1 \mathrm{~g}$ of organic matter which means that $70 \%$ of the sample is only organic matter.

In general, organo-soluble fractions (ORG) contain more carbon than water-soluble fractions (AQU). As we can see on Figure 2, the yield of organic carbon and nitrogen is higher towards more weakly bound fractions and that AQU2 and AQU3 fractions almost do not contain neither one of them. Overall, we were only able to extract around $2.4 \%$ of total organic carbon and slightly more, around $4.6 \%$, of total nitrogen contained. Both yields are relatively small so it can be deduced that the investigated biochar has a solid structure that does not 
allow us to extract much of its organic content. The alkaline extraction has even lower yields - about $0.9 \%$ of carbon and $1.2 \%$ of nitrogen.

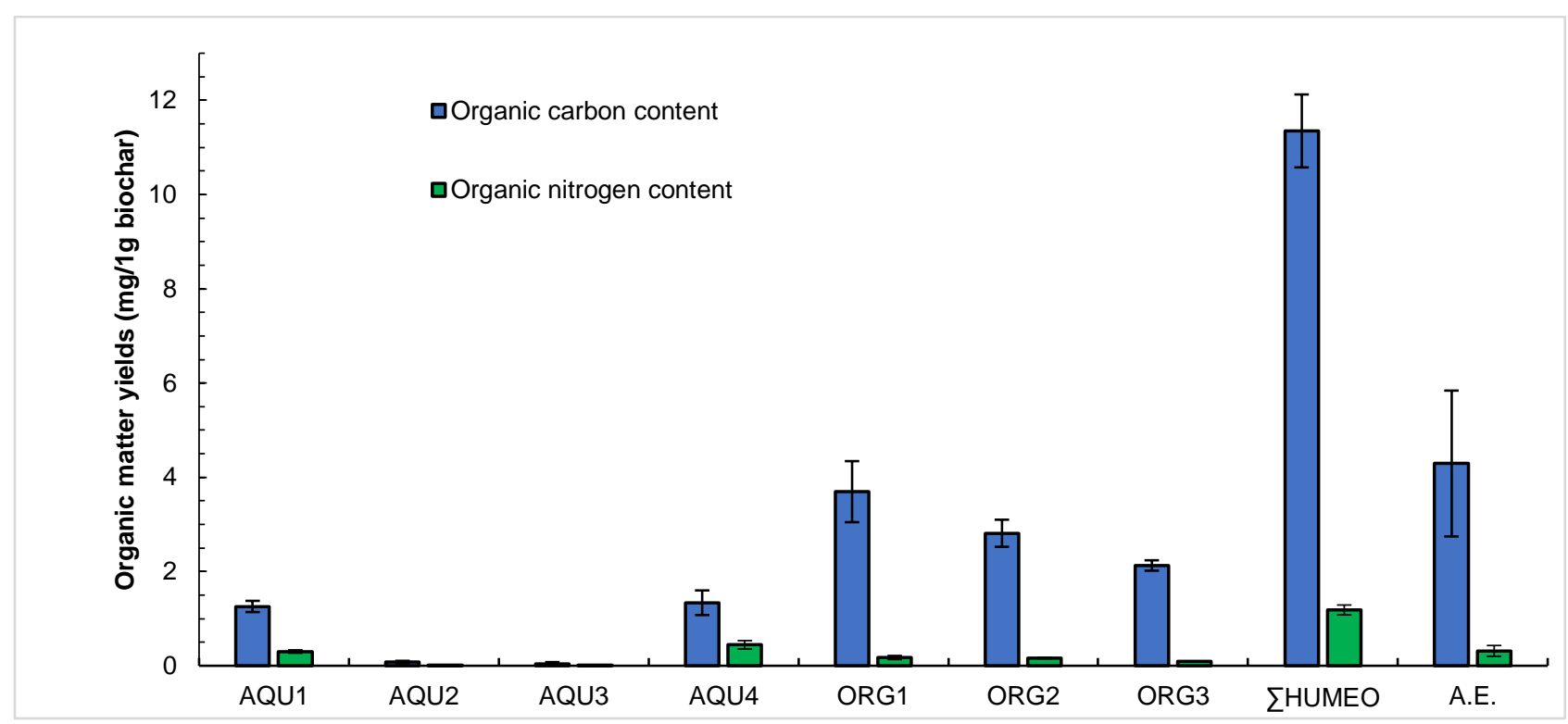

Figure 2 Organic matter content. The blue color represents the organic carbon content and the green color represents the organic nitrogen content.

So, as we can see above on Figure 2, the total amount of isolated organic carbon was about $12 \mathrm{mg}$ per $1 \mathrm{~g}$ of source biochar which is really a low amount considering that it contains $(476 \pm 50) \mathrm{mg}$ per $1 \mathrm{~g}$. Nevertheless, it was much more than we were able to isolate by the alkaline extraction which makes the sequential chemical fractionation more appropriate method to use. It is more laborious but also more than twice as effective as alkaline extraction. In case of nitrogen it is more than 4 times as effective. Similar facts can be found in the work of Drosos et. al. [7]. Moreover, separating the extracted organic matter into individual fractions allows us to more easily characterize those fractions using advanced spectroscopic and chromatographic techniques which provides us with more comprehensive information about the biochar sample and will be important for us to predict its effect on soil after its application into the soil.

\section{CONCLUSION}

This contribution was focused on describing the organic matter of biochar in order to better understand the effect of its use as a soil supplement. The biochar was first fractionated using the sequential chemical fractionation. Fractions containing organic matter with different solubilities (water and organo-soluble) were prepared and the individual fractions were separated based on their binding force to the residual biochar matrix. The other method used was alkaline extraction. All the samples were subsequently characterized by elemental analysis which determined the organic elements (carbon, hydrogen, nitrogen and sulfur) content and, in order to determine the total organic and inorganic matter content, the thermogravimetric analysis was used.

The results showed how much and which organic elements are contained in the biochar sample related to $1 \mathrm{~g}$ of it. The assumptions about the solid structure were confirmed according to the fact that, despite the use of strong and relatively aggressive solvents, it has been impossible for us to extract a significant amount of total organic matter contained. Nevertheless, the biochar has a great potential for use in agricultural sector as a soil supplement as biochar is likely to be retained in the soil matrix for a relatively long time and thus contribute to the stabilization of soil organic matter. 
Overall, the analyzes show that the investigated biochar is a really rigid and durable material that, even after using of aggressive reagents and high temperatures, still contains a high amount of organic matter. The structure couldn't be cleaved at a level where strongly bound organic matter could be extracted, which is indicating the high resistance of organic matter in biochar structure.

To be able to make more detailed description we will perform chromatographic analysis (GC-MS and LC-MS) which will provide us information on the molecular level of fractions or structural analysis methods (e.g. FTIR, NMR). Another follow-up research will include using these separation methods to characterize a biochar that has been cultivated and treated with soil microorganisms. That will make it possible to better assess their potential effect on biochar in the soil. This experimental set-up will bring crucial insights into the study on the bioavailability of organic matter of biochar for the surrounding soil environment.

\section{ACKNOWLEDGEMENTS}

\section{This work has been supported by project GJ20-28208Y from Czech Science Foundation of the Czech} Republic.

\section{REFERENCES}

[1] Agronomy Fact Sheet no. 41: Soil organic matter. Cornell University Publications, 2008. [cit. 2014-01-21].

[2] PAUL, E. A., CLARK, F. E. Soil Microbiology and Biochemistry. San Diego: Academic Press, 1996, pp. 93, 320.

[3] TAN, K.H. Humic Matter in Soil and the Environment. Principles and Controversies. New York: Marcel Dekker, Inc., 2003, p. 386.

[4] GUSTAFSON, A. F. Soils and soil management. London: McGraw-Hill Book Company, 1941. McGraw-Hill publications in the agricultural sciences.

[5] BOHLEN, P. J.; EDWARDS, C. A. Earthworm effects on $\mathrm{N}$ dynamics and soil respiration in microcosms receiving organic and inorganic nutrients. Soil Biology and 50 Biochemistry. 1995, vol. 27, pp. 341-348. [cit. 2017-02-26].

[6] NEBBIOSO, A., PICCOLO, A. Basis of a Humeomics Science: Chemical Fractionation and Molecular Characterization of Humic Biosuprastructures. Biomacromolecules. 2011, vol. 12, no. 4, pp. 1187-1199. [cit. 2019-03-31].

[7] DROSOS, M., NEBBIOSO, A., MAZZEI, P., VINCI, G., SPACCINI, R., PICCOLO, A. A molecular zoom into soil Humeome by a direct sequential chemical fractionation of soil. Science of The Total Environment. 2017, vol. 586, pp. 807-816.

[8] MASTO, R. ebhin, Md. a. ANSARI, J. GEORGE, V.a. SELVI a L.c. RAM. Co-application of biochar and lignite fly ash on soil nutrients and biological parameters at 77 different crop growth stages of Zea mays. Ecological Engineering. Elsevier B.V. 2013, vol. 58, pp. 314-322. [cit. 2019-04-05].

[9] RAM, L., SRIVASTAVA, N., JHA, S., SINHA, A., MASTO, R., SELVI, V. Management of Lignite Fly Ash for Improving Soil Fertility and Crop Productivity. Environmental Management. New York: Springer-Verlag, 2007, vol. 40, no. 3, pp. 438-452. [cit. 2019-04-07].

[10] LEHMANN, Johannes, RILLIG, Matthias C., THIES, Janice, MASIELLO, Caroline A., HOCKADAY, William C. and CROWLEY, David. Biochar effects on soil biota - A review. Soil Biology and Biochemistry. [online]. 2011, vol. 43, no. 9, pp. 1812-1836. Available from: https://doi.org/10.1016/i.soilbio.2011.04.022. ISSN 00380717.

[11] TAN, Zhongxin, LIN, Carol S.K., JIn Xiaoyan, RAINEY, Thomas J. Returning biochar to fields: A review. Applied Soil Ecology. [online]. 2017, vol. 116, pp. 1-11. Available from: https://doi.org/10.1016/j.apsoil.2017.03.017. ISSN 09291393.

[12] ŠIRŮČEK, David, KALINA Michal, KLUČÁKOVÁ Martina. Influence of the Application of lignite on the distribution of organic carbon in soil. In: NANOCON 2020 12th International Conference on Nanomaterials - Research and Application. [online]. Ostrava: TANGER, 2020, p. 489-494. ISBN 978-80-87294-98-7. ISSN 2694-930X. Available from: https://doi.org/10.37904/nanocon.2020.3765 